\title{
A morphometric analysis of nucleoli in cultured carcinoma cells of the human thyroid
}

\author{
Lars Andersen ${ }^{\mathrm{a}, *}$, Lars Kayser ${ }^{\mathrm{a}}$, Niels Keiding ${ }^{\mathrm{b}}$ and Jens Thomsen ${ }^{\mathrm{c}}$ \\ ${ }^{\mathrm{a}}$ Department of Medical Anatomy and ${ }^{\mathrm{b}}$ Department of Biostatistics, University of Copenhagen, \\ Copenhagen, Denmark \\ ${ }^{\mathrm{c}}$ Department of Surgery, Copenhagen County Hospital Gentofte, Copenhagen, Denmark
}

\begin{abstract}
Cells from 7 patients operated on for thyroid cancer were investigated. Samples of cells from the carcinoma and from the normal thyroid tissue were cultured with and without TSH stimulation. For light microscopy, serial sections of cells were cut and the size of nucleoli was measured and the number of nucleoli per cell counted. At the electron microscopic level the number and the volume of the fibrillar centres (FC) were estimated taking the Swiss cheese effect into account. The areal densities of FC, the fibrillar and granular component in nucleoli were determined by point counting. The results indicate that the malignant transformation has no influence on the size of the FC, but the observed numbers as well as the total area of FC are larger in cancer cells than in the normal thyroid epithelial cells. The nucleolar density of the fibrillar component is larger and that of the granular component is smaller in thyroid carcinoma cells than in non-malignant thyroid epithelial cells $(p=0.0001)$. Thus simple morphometry at the electron microscopic level might be helpful to discriminate between thyroid epithelial cells and thyroid carcinoma cells in culture.
\end{abstract}

Keywords: Thyroid gland, nucleoli, fibrillar centres, culture, cancer

\section{Introduction}

Nucleolar organizer associated proteins (NORAPs) are essential for ribosomal gene transcription and have been recognised in the nucleolar organizer regions (NORs). They are of fundamental interest to tumour cell biologists [4]. Accumulations of the NORAP's are visualized as argyrophil components of nucleoli (AgNOR) in the light microscope and as fibrillar centres (FC) in the electron microscope. Although the AgNOR reaction occurs mainly in the nucleolar fibrillar centres [3], the fibrillar component of the nucleoli also shows AgNOR reaction [6]. The numbers of discernible (and therefore countable) interphase AgNOR dots depend on at least three variables: the degree of NOR dispersion, the spatial resolution of the optical system (microscope coupled to either image analyser or human observer) and control of the AgNOR staining technique [22]. AgNOR profile areas have been measured using image cytometry [19], but the resolution of the image analysis systems is too low to recognize the individual FC. Moreover, the AgNOR dots lie at different levels in the nuclear section

\footnotetext{
${ }^{*}$ Corresponding author. Department of Medical Anatomy, The Panum Institute, Blegdamsvej 3, DK-2200 Copenhagen N, Denmark. Tel.: +45 3532 7219; Fax: +45 3532 7217; E-mail: L.Andersen@mai.ku.dk.
} 
decreasing the resolution of individual dots. At the electron microscopic level the number and volume of FC can be estimated without these shortcomings [2]. It has been demonstrated that the degree of differentiation, rather than the proliferation state, may sometimes be related to the AgNOR numbers per nucleus [8]. The primary objective of the present investigation is to determine whether the size and number of NORAP accumulations in malignant cells differed from those of non-malignant cells. In normal cells, stimulation of the secretion influences the content of the nucleolar components $[1,9,20]$. The secondary objective was to investigate to what extent the content of the fibrillar and granular components differs between thyroid epithelial cells (TEC) and thyroid carcinoma cells (TCC) and the influence of TSH on both sets of samples. The nucleoli were examined at the light as well as the electron microscopic level.

\section{Material and methods}

\subsection{Culture and stimulation}

TCC and TEC were obtained in connection with strumectomies of 7 patients with thyroid cancer and prepared as previously described [18]. Diagnostic criteria: the criteria of the World Health Organization were used [12]. The cells were cultured in Hams F12 medium modified by Kohn [16] supplemented with 5\% fetal calf serum (Biological Industries), antibiotics (100 kU/l penicillin, $100 \mathrm{mg} / \mathrm{l}$ streptomycin, Biological Industries) and six growth factors: bovine TSH 1 U/l (Armour, Collegeville, PA, USA), human insulin $10 \mathrm{mg} / \mathrm{l}$ (Boehringer, Mannheim, Germany), somatostatin $10 \mu \mathrm{g} / \mathrm{l}$ (Novabiochem, Länfalfingen, Switzerland), human transferrin $6 \mathrm{mg} / \mathrm{l}$ (Boehringer), hydrocortisone $10^{-8} \mathrm{M}$ (Calbiochem, La Jolla, CA, USA), glycyl-histidyl-lysine acetate $10 \mu \mathrm{g} / \mathrm{l}$ (Calbiochem) and nonessential amino acids (Biological Industries). After one week the cells were passaged and seeded into 4 TEC-25 plastic flasks. Two of the TEC-25 flasks were stimulated with TSH for $72 \mathrm{~h}$. This resulted in four samples of cells from each patient. Two samples of TCC from the carcinoma, one TSH-stimulated $\left(\mathrm{TCC}^{+}\right)$and one unstimulated $\left(\mathrm{TCC}^{-}\right)$. TEC were obtained from apparently normal thyroid tissue situated in the opposite site of the carcinoma, one TSH-stimulated culture $\left(\mathrm{TEC}^{+}\right)$and one unstimulated $\left(\mathrm{TEC}^{-}\right)$. A total number of 7 patients were investigated. In 5 patients we were able to obtain and grow cells from normal and malignant tissue. Only in three patients a complete set of TEC and TCC, grown with and without TSH, was obtained.

\subsection{Fixation and embedding}

After a wash in Hanks BBS, the cells were fixed in $2 \%$ glutaraldehyde (TAAB) in $0.1 \mathrm{M}$ cacodylate buffer ( $\mathrm{pH}$ 7.0) for $2 \mathrm{~h}$ at room temperature. The cells were then loosened mechanically with a plastic scraper, transferred to tubes and centrifuged for $5 \mathrm{~min}$ at $1700 \mathrm{rpm}$. The sediment was transferred to $2 \%$ glutaraldehyde in $0.1 \mathrm{M}$ cacodylate buffer and kept overnight at room temperature. The cells were postfixed in $2 \% \mathrm{OsO}_{4}$ in $0.1 \mathrm{M}$ cacodylate buffer for $2 \mathrm{~h}$, rinsed very briefly in $0.1 \%$ cacodylate buffer and in distilled water, dehydrated in 70, 96 and 99\% ethanol, and routinely embedded in Epon for light and electron microscopy.

\subsection{Microscopy and morphometry}

The microscopy and the morphometry were performed with the observer unaware of the identity of the sample involved. For light microscopy, consecutive $1 \mu \mathrm{m}$ thick sections were cut from each sample 
and stained with toluidine blue until 500 nuclei were fully sectioned. The sections were examined using a Leitz Orthoplan microscope equipped with a $100 \times$ Plan Apo objective (NA 1,32) attached to a video camera Kappa CF 11/2. The number of nucleoli per nucleus was counted in sections observed on a TV-screen (final magnification of 4,500×). Nucleoli were defined as round intranuclear bodies with an average diameter $>1 \mu \mathrm{m}$. The diameter of the nucleolar profile was measured with a set of test circles of increasing diameter on a transparency. The circle which fitted best with the largest profile size was chosen. The nucleoli were assumed to be spheres, and the individual nucleolar volumes were calculated using the formula $V=(1 / 6) \pi D^{3}$. The means of the following parameters were calculated for 20 nuclei in each sample: the number of nucleoli per nucleus, the volume of the individual nucleoli and the volume of the nucleoli per cell. The chosen nuclei were the first 20 nuclei not seen in the first section. To follow these 20 nuclei through the serial sections photographs were taken of every third section and the individual nuclei were identified by means of pattern recognition of the shape of the nucleus and the cell as well as the position in relation to the other cells. Furthermore, the metaphase index (MI) was determined as the ratio of cells arrested in metaphase to the total number of cells counted (500) [7].

For electron microscopy, $70 \mathrm{~nm}$ thick sections were cut from several levels at $4 \mu \mathrm{m}$ intervals. $70 \mathrm{~nm}$ was the minimal thickness which provided enough contrast to identify the FC. All nucleoli from each level were photographed until a total of at least 55 nucleoli from each specimen was reached, resulting in a total of 1300 pictures. The following criteria were used for identification of the nucleolar compartments. Fibrillar centres: small rounded areas containing fibrils about $5 \mathrm{~nm}$ in diameter and displaying a low electron density [11]. Fibrillar component: dense osmiophilic material composed of fibrils with diameters in the range of 4-10 nm [11]. Similar fibrils of lower electron density situated in or near interstices were also categorized as fibrillar component. Granular component: areas containing granules $15-20 \mathrm{~nm}$ in diameter. Interstices: light areas of lower electron density than the surrounding nucleolar mass [10]. Micrographs with a final magnification of $42,000 \times$ were examined through a magnifying glass with a magnification factor of 2 . A quadratic test lattice with $2.0 \mathrm{~cm}$ spacing was superimposed on each micrograph and the densities of the FC, the fibrillar and granular components as well as the interstices were determined. The profile diameter of the FC was measured with a transparent set of test circles of increasing diameter. The circle which fitted best with the profile size of the FC was chosen. The FC appeared as small rounded areas of low electron density and were surrounded by the fibrillar component of high electron density. When tissue sections are observed in the electron microscope, the FC appear as holes in a Swiss cheese. A slice of cheese will show a profile of a hole only when each of the two planar faces of the slice penetrate the same hole. Moreover, the diameter of the profile will be smaller than the diameter of the hole. The diameters of the FC $\left(D_{\mathrm{e}}\right)$ were estimated from the observed diameters $\left(D_{\mathrm{o}}\right)$ taking the Swiss cheese effect into account by a modification of the method described by Keiding and Andersen [14]; instead of the earlier used $\chi^{2}$-distributions, logarithmically normal distributions of true diameters were used for increased flexibility [15]. The number of FC per observed square micrometer $\left(N_{A}\right)$ was calculated, after which the numerical density in nucleoli

$$
N_{V}=N_{A} /\left(D_{\mathrm{e}} \cos \Theta-T\right)
$$

could be determined. $\Theta$ is the minimal size of the capping angle between the section and the FC, necessary to identify FC. The mean area $(\bar{A})$ and the mean volume $(\bar{V})$ of the individual FC were calculated using the formulas $A=D^{2} \pi / 4$ and $V=D^{3} \pi / 6$, where $D^{2}$ and $D^{3}$ were estimated from the fitted FC diameter distributions. The volume of FC per cell is calculated by multiplying $N_{V}$ by $\bar{V}$. 


\subsection{Statistics}

For each variable there were measurements from 7 patients on some or all of the samples $\mathrm{TEC}^{-}$, $\mathrm{TEC}^{+}, \mathrm{TCC}^{-}, \mathrm{TCC}^{+}$. The comparisons between samples had to take the individual matching into account and were performed as an unbalanced two-way analysis of variance except for the nucleolar densities for which the counts were analysed in a logistic regression analysis based on the same layout. All $p$-values quoted are from these analyses, so when comparing, e.g., $\mathrm{TEC}^{-}$and $\mathrm{TCC}^{-}$, the $p$-value for the contrast between these two factors in the analysis of variance is quoted.

\section{Results}

Light microscopy showed that the observed cells contained one nucleus and all nuclei contained at least one nucleolus, the average of all samples being $1.98 \pm 0.45$ nucleolus per cell. For each patient, the numbers and volumes of nucleoli in the $\mathrm{TEC}^{-}$and $\mathrm{TCC}^{-}$samples are shown in Fig. 1. The number of nucleoli in $\mathrm{TEC}^{-}$is larger than in $\mathrm{TCC}^{-}(p=0.039)$. The volumes of the individual nucleoli varied from patient to patient, but there was no significant pattern in the variations of the nucleolar volumes. When the $\mathrm{TEC}^{+}$and $\mathrm{TCC}^{+}$samples were compared, there was no change in the number of nucleoli per cell, nor was there any systematic change in the volume of nucleoli per cell. These results are not shown in the figures. In the TEC and TCC samples cultured with and without TSH the MI was maximally $2 \%$ and there was no difference between the TEC and TCC samples.

In the electron microscope, FC appear as light circular areas in the nucleoli and it is easy to distinguish FC, the fibrillar and granular compartments from each other (Fig. 2). The diameters of the FC were measured and estimated. The distribution of these diameters in one of the $\mathrm{TCC}^{-}$samples is shown in Fig. 3. The differences between $D_{\mathrm{o}}$ and $D_{\mathrm{e}}$ are small. The difference between the means of $D_{\mathrm{o}}$ and $D_{\mathrm{e}}$ is a few nanometers. Only in one patient was the difference between the means about $20 \mathrm{~nm}$. For each patient, the observed diameters of FC in the unstimulated cells are compared in Fig. 4(A), the means show no significant difference $(p=0.19)$.
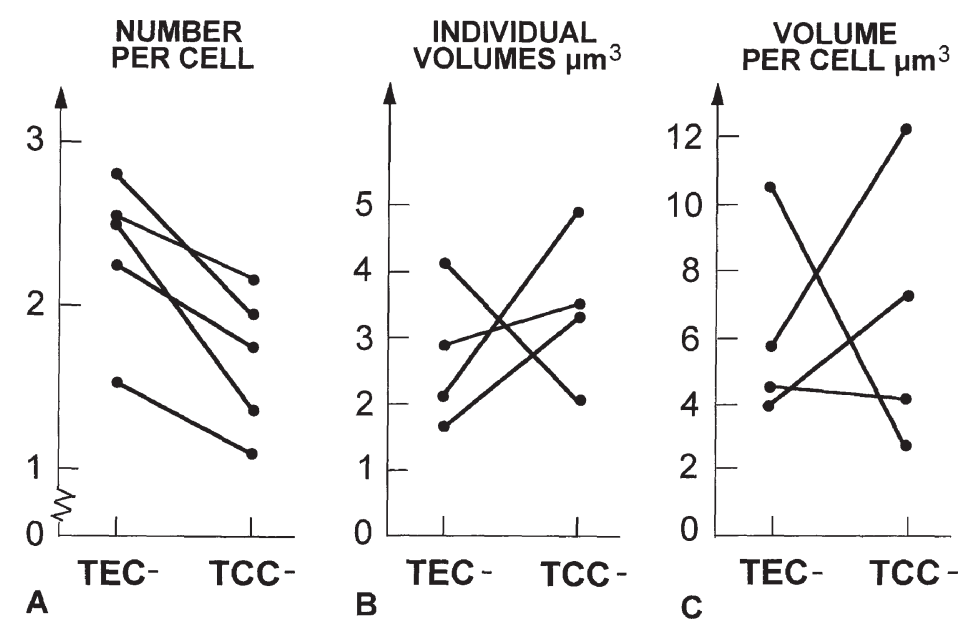

Fig. 1. Pairs of mean numbers and mean volumes of nucleoli per cell in thyroid epithelial cells (TEC) and thyroid carcinoma cells (TCC) grown without TSH (-) from each patient. 


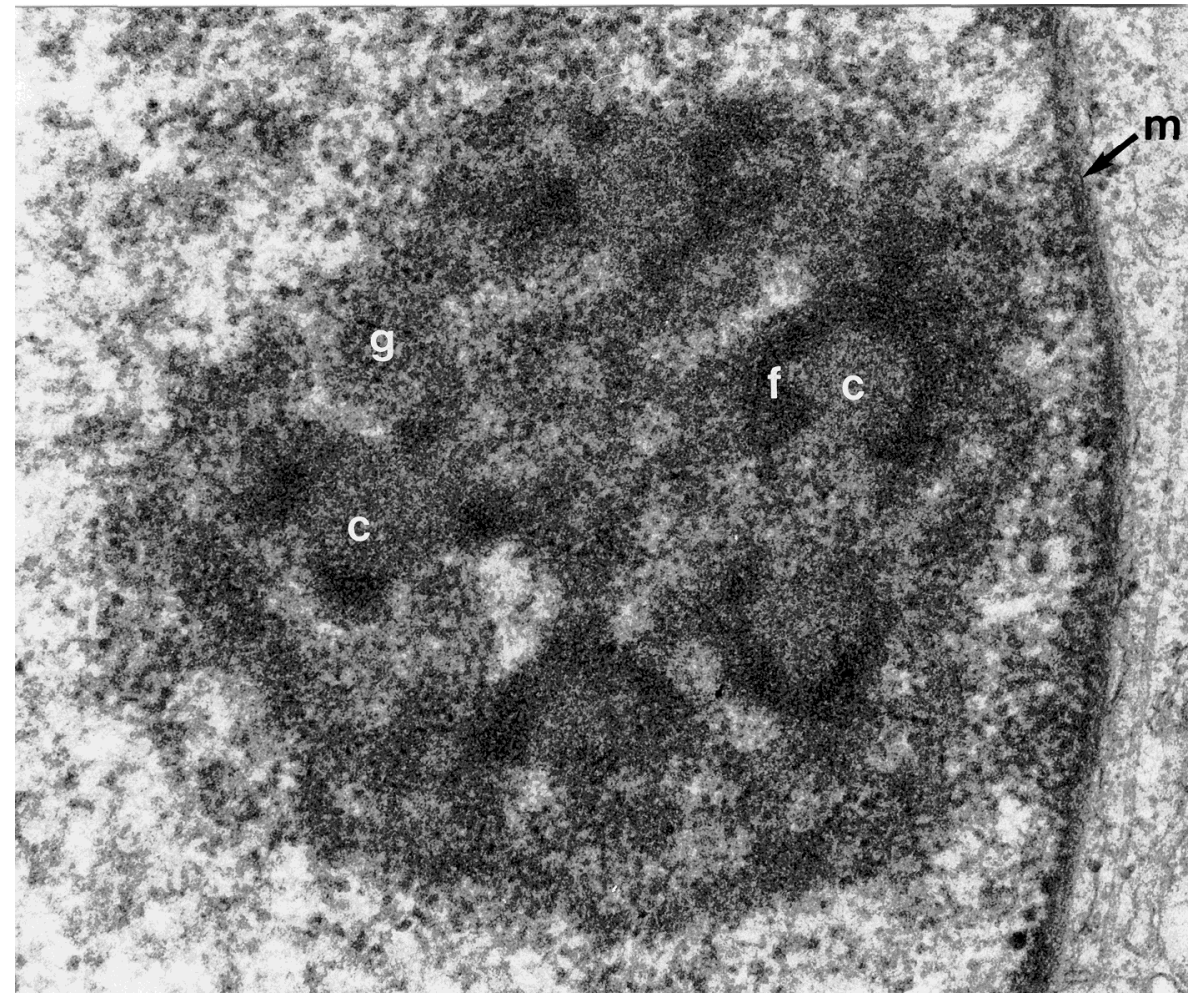

Fig. 2. Nucleolus of thyroid carcinoma cells grown without TSH. The fibrillar centres (c) are sharply delineated from the surrounding fibrillar component (f). Moreover, the granular component $(\mathrm{g})$ and the nuclear membrane $(\mathrm{m})$ are indicated $(\times 46,000)$

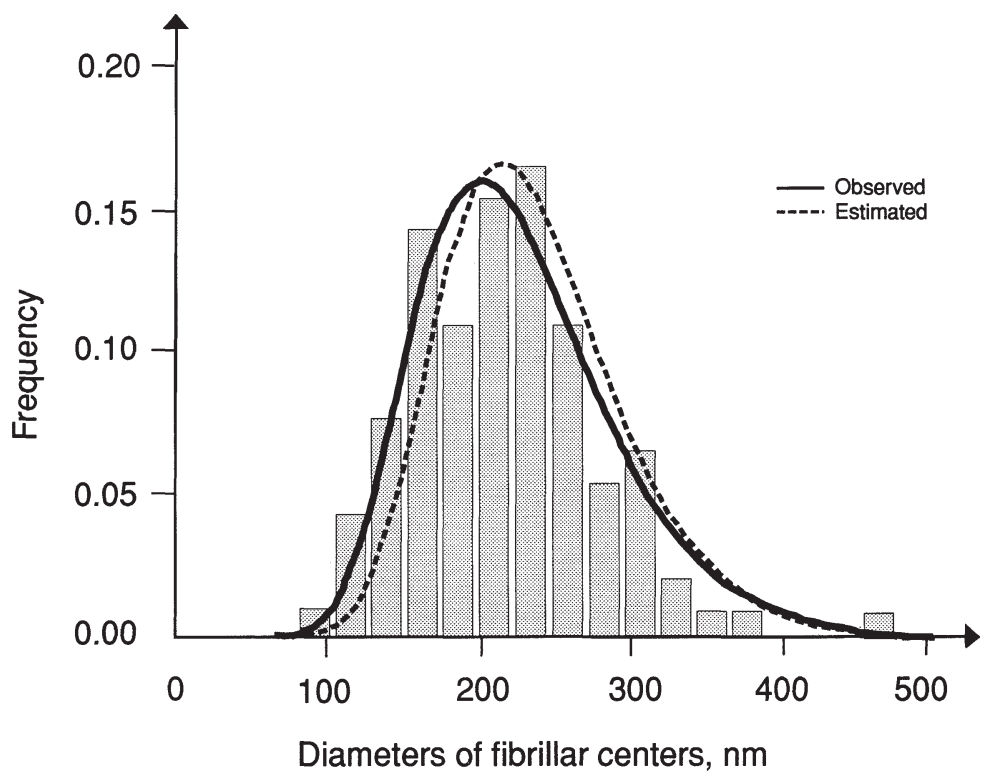

Fig. 3. Size distributions of diameters of fibrillar centres in one sample of carcinoma cells grown without TSH. The observed diameters, and the estimated diameters are shown taking the Swiss cheese effect into account. 

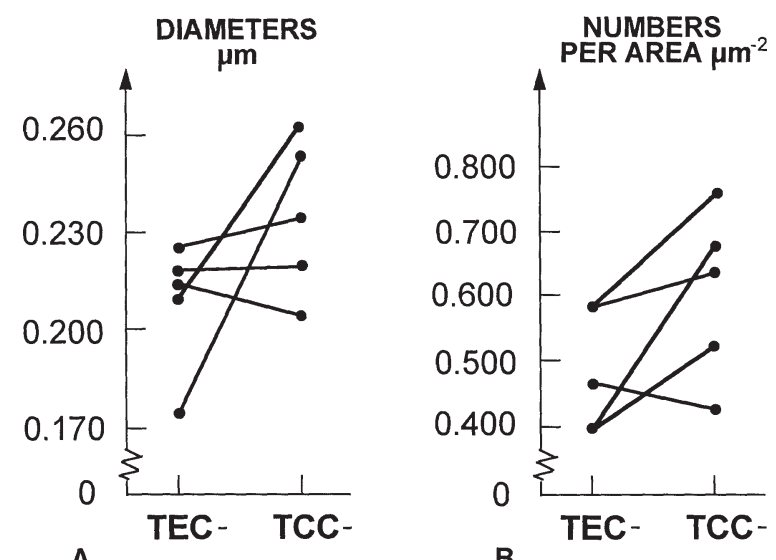

Fig. 4. Pairs of observed diameters and numbers of fibrillar centres in nucleoli from each patient. The means in thyroid epithelial cells (TEC) and thyroid carcinoma cells (TCC) grown without TSH (-).
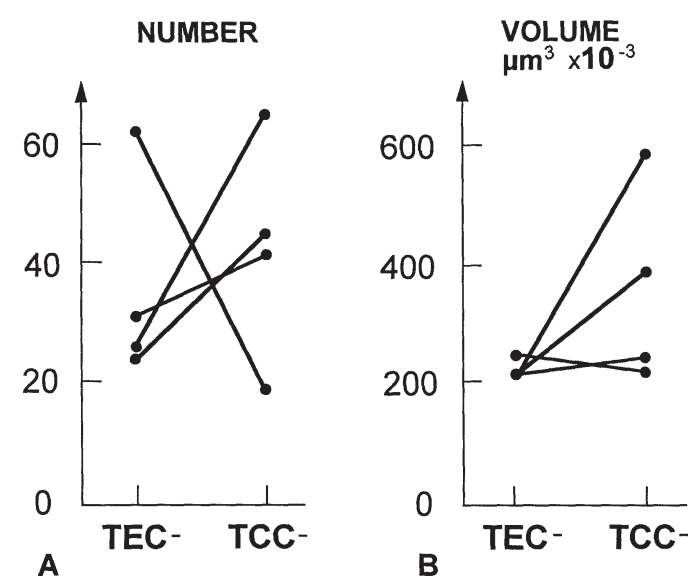

Fig. 5. Pairs of means of number and volume of fibrillar centres per cell from each patient. These parameters are estimated taking the Swiss cheese effect into account.

The observed numbers of FC per nucleolar area in TCC and TEC from each patient were compared. The results for the unstimulated samples are shown in Fig. 4(B). The means of the numbers in $\mathrm{TCC}^{-}$ are larger than in $\mathrm{TEC}^{-}(p=0.044)$. In contrast, the numbers of $\mathrm{FC}$ in the stimulated $\mathrm{TEC}^{+}$and $\mathrm{TCC}^{+}$samples are nearly the same. Only in three out of 5 patients is it possible to compare the influence of TSH on both non-malignant and malignant cells. In two of these three patients the number of FC is larger in malignant than in non-malignant cells.

The estimated numbers and volumes of FC per cell in the unstimulated cells are shown in Fig. 5. There are no significant differences between the numbers of FC per cell $(p=0.44)$, but the volumes of FC per cell tend to be larger in the $\mathrm{TCC}^{-}$than in the $\mathrm{TEC}^{-}$samples $(p=0.18)$. No differences can be demonstrated between the stimulated cells.

The nucleolar densities of the FC, the fibrillar component and the granular component in the unstimulated cells are presented in Fig. 6. Whereas the density of FC is larger in $\mathrm{TCC}^{-}$than in $\mathrm{TEC}^{-}$ $(p=0.009)$, there is no significant difference between $\mathrm{TCC}^{+}$and $\mathrm{TEC}^{+}$. The fibrillar component makes up a larger proportion of the nucleoli in $\mathrm{TCC}^{-}$than in $\mathrm{TEC}^{-}(p=0.0002)$ and correspondingly 

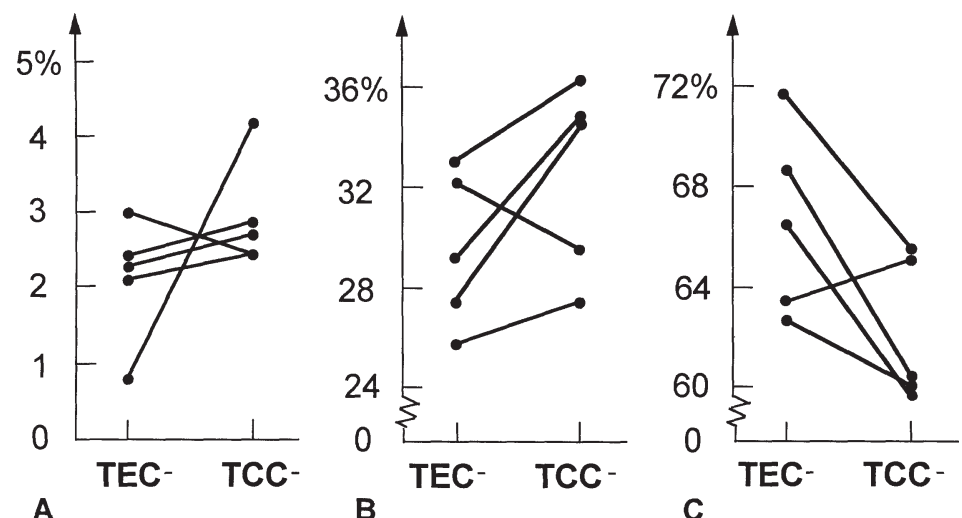

Fig. 6. The areal densities of centres, fibrillar and granular components in nucleoli in cells grown without TSH (-). Pairs of the means in thyroid epithelial cells (TEC) and thyroid carcinoma cells (TCC) grown without TSH from each patient.

CENTERS

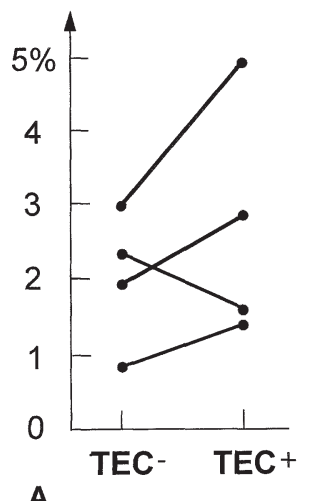

FIBRILLAR

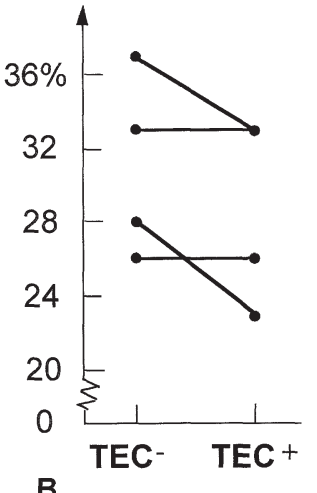

GRANULAR

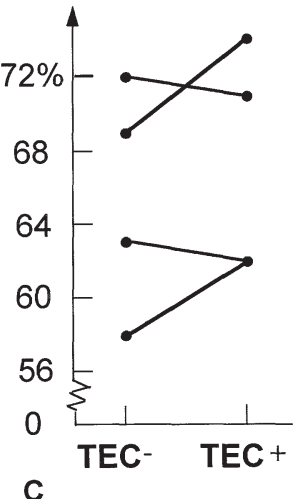

Fig. 7. The areal densities of centres, fibrillar and granular components in nucleoli of thyroid epithelial cells (TEC) grown with (+) and without (-) TSH. Pairs from each patient are compared.

the granular component makes up a lesser part in $\mathrm{TCC}^{-}$than in $\mathrm{TEC}^{-}(p=0.0001)$. When comparing $\mathrm{TCC}^{+}$with $\mathrm{TEC}^{+}$, the densities of the fibrillar and granular components show the same pattern $(p=0.0001)$.

The unstimulated and stimulated TEC are compared in Fig. 7. The fibrillar component has a larger density in $\mathrm{TEC}^{-}$than in $\mathrm{TEC}^{+}(p=0.008)$ and the granular component has a smaller density in $\mathrm{TEC}^{-}$than in $\mathrm{TEC}^{+}(p=0.058)$. There is no significant difference between the densities of the FC. A comparison of $\mathrm{TCC}^{-}$and $\mathrm{TCC}^{+}$shows no detectable differences in the densities of the fibrillar component or the granular component or FC.

\section{Discussion}

By studying serial sections of the glutaraldehyde fixed samples, nucleoli were found in all cells, TEC as well as TCC. Montironi et al. [17] studied cell smears obtained from fine needle aspirations. 
They found nuclei, which contained nucleoli in $50 \%$ of the follicular adenoma cells and $74 \%$ of the follicular carcinoma cells. Montironi found a higher percentage of cells with two or more nucleoli in carcinoma cells than in normal cells. In cells cultured with TSH we found an equal number of nucleoli in carcinoma and normal cells. But in cells cultured without TSH the number of nucleoli in normal cells was larger than in cancer cells. The overall average number of nucleoli was 1.98, which may indicate that our method is more sensitive. However, these discrepancies may also be due to differences in obtaining the specimens, e.g., secondary cultures versus fine needle aspiration, fixation methods and/or the counting technique.

The mean volume of the nucleoli per cell was measured and there was no significant difference between the samples. Hence, under our experimental conditions, changes in volume densities of the nucleolar compartments reflect the changes in the total content per cell. As mentioned above, there were fewer nucleoli in $\mathrm{TCC}^{-}$than in $\mathrm{TEC}^{-}$, but the volumes of the individual nucleoli did not show an opposite significant pattern to compensate for the change in number. Therefore the result that the volume per cell is identical in the samples should be interpreted with caution.

The difference between the observed and estimated diameters of the FC is smaller than in earlier observations of neurons in the rat [2]. The size distribution found in this study makes the difference smaller; so small that it is acceptable to disregard the Swiss cheese effect in future studies of TEC and TCC.

AgNORs are the product of staining argyrophilic non-histone proteins which are mostly comprised of two NORAPs, nucleolin $\left(\mathrm{C}_{22}\right)$ and numatrin $\left(\mathrm{B}_{23}\right)$ [13]. If these argyrophilic NORAPs are mainly situated in the FC [3] three results of our morphometric investigations of FC support the evaluation of AgNORs as a means in cancer diagnostics. But if a considerable part of the argyrophilic NORAPs are localized in the fibrillar component the comparisons between FC and AgNOR carry less weight. First, there was no significant difference between the diameters of FC in the $\mathrm{TEC}^{-}$and $\mathrm{TCC}^{-}$samples. Thus, an AgNOR staining should give the same proportion of discernible AgNOR dots. Second, the numerical density of $\mathrm{FC}$ in the nucleoli was larger in $\mathrm{TCC}^{-}$than in $\mathrm{TEC}^{-}$. As already stated, the size of the nucleoli in these two categories of samples is the same. Therefore, in AgNOR stained samples the number of discernible AgNOR dots per nucleolus should be larger in the $\mathrm{TCC}^{-}$than in the $\mathrm{TEC}^{-}$. Third, the areal density of FC in nucleoli was significantly larger in $\mathrm{TCC}^{-}$than in $\mathrm{TEC}^{-}$. This indicates that the areal density of AgNORs should be larger in $\mathrm{TCC}^{-}$than in $\mathrm{TEC}^{-}$.

A higly significant correlation between the AgNOR number and the speed of cell replication has been found in a series of experiments carried out on human cultured cancer cell lines [21]. In the present investigation the percentage of cells in division was the same in TEC and TCC. Thus, the observed increased number of $\mathrm{FC}$ in $\mathrm{TCC}^{-}$may be related to the transformation to cancer and to the degree of differentiation. This is in accordance with Edwards et al. [8] who demonstrate that in a promonocytic cell line the degree of differentiation rather than the proliferation state may be related to AgNOR numbers. Derenzini [5] proposed that cells capable of dividing rapidly must concentrate their ribosomal biogenesis in a shorter time. This can be achieved by activating a greater number of rDNA sequences for transcription. Therefore a greater number of NORAPs must be synthesized, which gives rise to a greater number of FC.

In TCC the volume density of the fibrillar component was higher and that of the granular component lower than in TEC. Thus a ratio between the fibrillar and granular densities might be a useful method for establishing a differential diagnosis between non-malignant and malignant cells in cultures. However, it remains to be shown in biopsies. TSH decreased the density of the fibrillar component and increased that of the granular component. These changes are seen in other cellular systems in which protein 
synthesis is stimulated $[1,9,20]$. Thyrocytes in secondary monolayer culture preserve the ability to produce thyroglobulin (TG) and to react to TSH-stimulation [18]. Thus the decrease in density of the fibrillar component and the increase of the granular component in the present investigation may be related to increased protein synthesis. When TCC were treated with TSH the densities of the components did not change. This indicates that TSH does not increase protein synthesis in TCC. This may either be due to a lack of functioning TSH receptors or to a constitutively acting TSH receptor.

The density of $\mathrm{FC}$ was larger in $\mathrm{TCC}^{-}$than in $\mathrm{TEC}^{-}$. This indicates a lower level of protein synthesis [1], just as the observed higher density of the fibrillar component and the lower of the granular component does. These observations indicate that TCC in culture have a lower level of protein synthesis than TEC and do not respond to TSH stimulation. One of the authors of this paper has previously demonstrated that by inhibiting protein synthesis the number of FC decreased to onethird but the size of the individual FC increased to the double. This resulted in a $60 \%$ higher areal density of FC. Moreover, the density of the granular component decreased $(p=0.04)$ [2]. In electron as well as in light microscopy the number of FC-AgNOR dots and areal density of FC-AgNORs are two different parameters.

\section{Conclusion}

The investigated cells were almost exclusively in interphase and the proliferation state probably the same in TEC and TCC. The number as well as the total area of FC are larger in TCC than in TEC. This result supports the hypothesis that the number of FC may be related to the degree of differentiation. The density of the fibrillar component in nucleoli is larger and that of the granular component is smaller in TCC than in non-malignant TEC. Thus, simple morphometry at the electron microscopic level might be helpful to discriminate between TEC and TCC in culture.

\section{Acknowledgements}

The computationally demanding implementation of the new stereological algorithm and most of the other statistical calculations were performed by Hansoo Kim.

\section{References}

[1] L. Andersen, Electron microscopy and morphometry of nucleoli in rat neurosecretory cells with stimulated and suppressed secretion, Acta Anat. 138 (1990), 220-223.

[2] L. Andersen and N. Keiding, Size of the fibrillar centres of the nucleoli in the supraoptic nucleus of the rat taking the Swiss cheese effect into account, Acta Anat. 138 (1990), 348-351.

[3] J. Crocker, in: Cell Proliferation in Lymphomas, J. Crocker, ed., Blackwell Scientific Publ., London, 1993.

[4] J. Crocker, The trials and tribulations of interphase AgNORs, J. Pathol. 175 (1995), 367-368.

[5] M. Derenzini, Rationale for the use of interphase AgNOR parameters in tumour pathology, Virchows Arch. 427 (1995), 323-341.

[6] M. Derenzini, M. Thiry and G. Goessens, Ultrastructural cytochemistry of the mammalian cell nucleolus, J. Histochem. Cytochem. 38 (1990), 1237-1256.

[7] P.A. Ealey, J.E. Emmerson, S.P. Bidey and N.J. Marshall, Thyrotropin stimulation of mitogenesis of the rat thyroid cell strain FRTL-5: A metaphase index assay for the detection of thyroid growth stimulations, J. Endocrin. 106 (1985), 203-210.

[8] S. Edwards, S. Afford and J. Crocker, The effect of inducing agents on the numbers of interphase fibrillar centers in the U937 promonocytic cell line, Exp. Cell Res. 194 (1991), 118-121. 
[9] S. Fakan and R. Deltour, Ultrastructural visualization of nucleolar organizer activity during early germination of Zea mays L., Exp. Cell Res. 135(2) (1981), 277-282.

[10] G. Goessens, Nucleolar structure (review), Int. Rev. Cytol. 87 (1984), 107-158.

[11] A.A. Hadjiolov, The Nucleolus and Ribosome Biogenesis, Springer, Wien, 1985.

[12] C.E. Hedinger, E.D. Williams and L.H. Sobin, in: International Histological Classification of Tumours, C.E. Hedinger, ed., Springer, Berlin, 1988.

[13] S.D. Jewel and C.R. Cordial, Silver staining of nucleolar organizer regions, J. Histotechnol. 19(3) (1996), 241-256.

[14] N. Keiding and L. Andersen, Estimation of the size distribution of fibrillar centres in nucleoli - An example of the Swiss cheese problem in stereology, Biometrics 48 (1992), 449-458.

[15] N. Keiding and L. Andersen, Estimation of the size distribution of fibrillar centres in nucleoli - An example of the 'Swiss cheese', Biometrics 52 (1996), 1164

[16] L.D. Kohn and W.A. Valente, in: FRTL-5 Today, F. Ambesi-Impiombato and H. Perrild, eds, Excerpta Medica, Amsterdam, 1989.

[17] R. Montironi, A. Braccischi, M. Scarpelli, G. Matera and R. Alberti, Value of quantitative nucleolar features in the preoperative cytological diagnosis of follicular neoplasias of the thyroid, J. Clin. Pathol. 44 (1991), 509-514.

[18] Å.K. Rasmussen, L. Kayser, H. Perrild, M. Brandt, K. Bech and U. Feldt-Rasmussen, Human thyroid epithelial cells cultured in monolayers. I. Decreased thyroglobulin and cAMP responce to TSH in 12-week-old secondary and tertiary cultures, Mol. Cell Endocrin. 116 (1996), 165-172.

[19] J. Ruschoff, C. Prasser, T. Cortez, H.M. Hohne, W. Hohenberger and F. Hofstadter, Diagnostic value of AgNOR staining in follicular cell neoplasms of the thyroid - Comparison of evaluation methods and nucleolar features, Am. J. Surg. Pathol. 17 (1993), 1281-1288.

[20] A.S. Stoykova, M.D. Dabeva, R.N. Dimova and A.A. Hadjiolov, Ribosome biogenesis and nucleolar ultrastructure in neuronal and oligodendroglial rat brain cells, J. Neurochem. 45(6) (1985), 1667-1676.

[21] D. Trere, Critical analysis of the methods commonly employed in the assessment of cell proliferation - Advantages of the NOR silver-staining technique in routine cyto-histopathology (guest editorial), Anal. Cell. Pathol. 5 (1993), $191-201$.

[22] J.C.E. Underwood, AgNOR measurements as indices of proliferation, ploidy and prognosis, J. Clin. Pathol. Clin. Mol. Pathol. 48 (1995), M239-M240. 


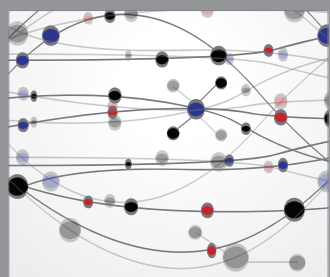

The Scientific World Journal
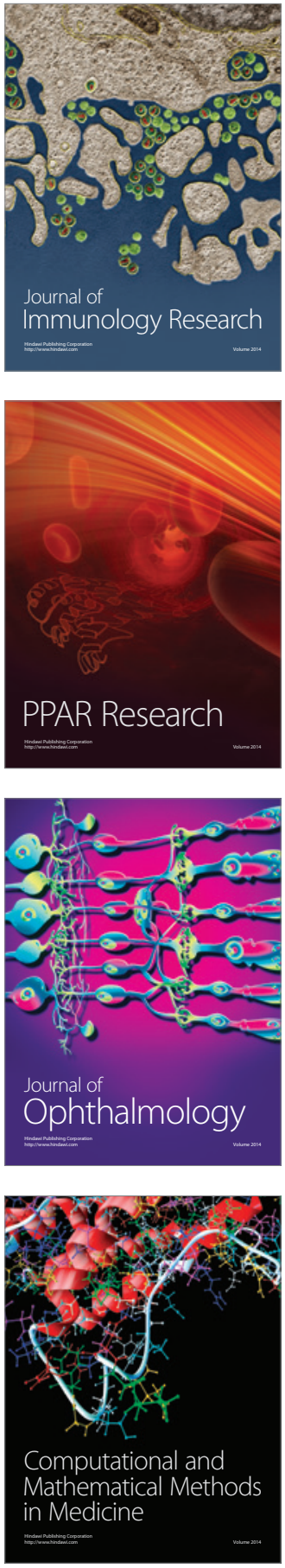

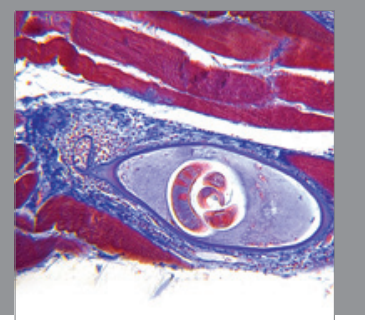

Gastroenterology

Research and Practice
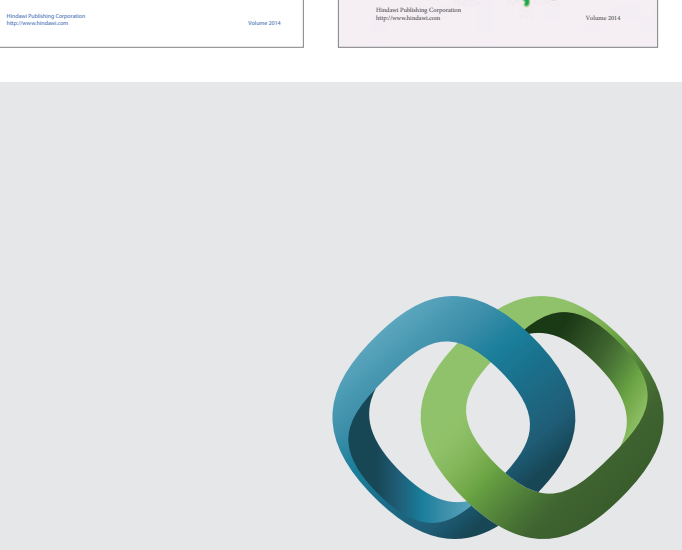

\section{Hindawi}

Submit your manuscripts at

http://www.hindawi.com
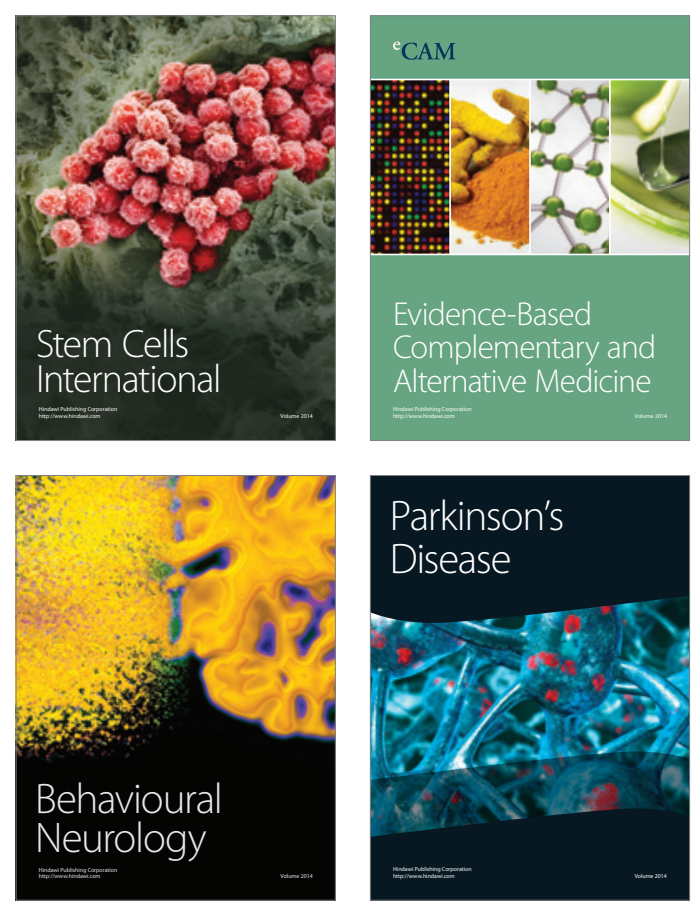

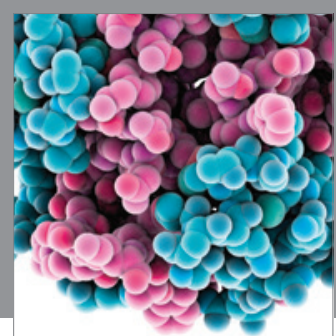

Journal of
Diabetes Research

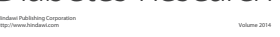

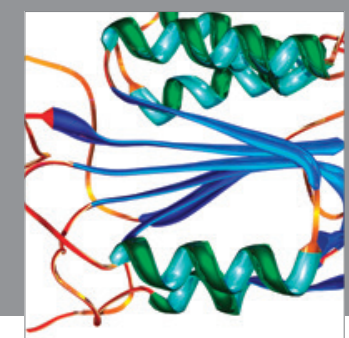

Disease Markers
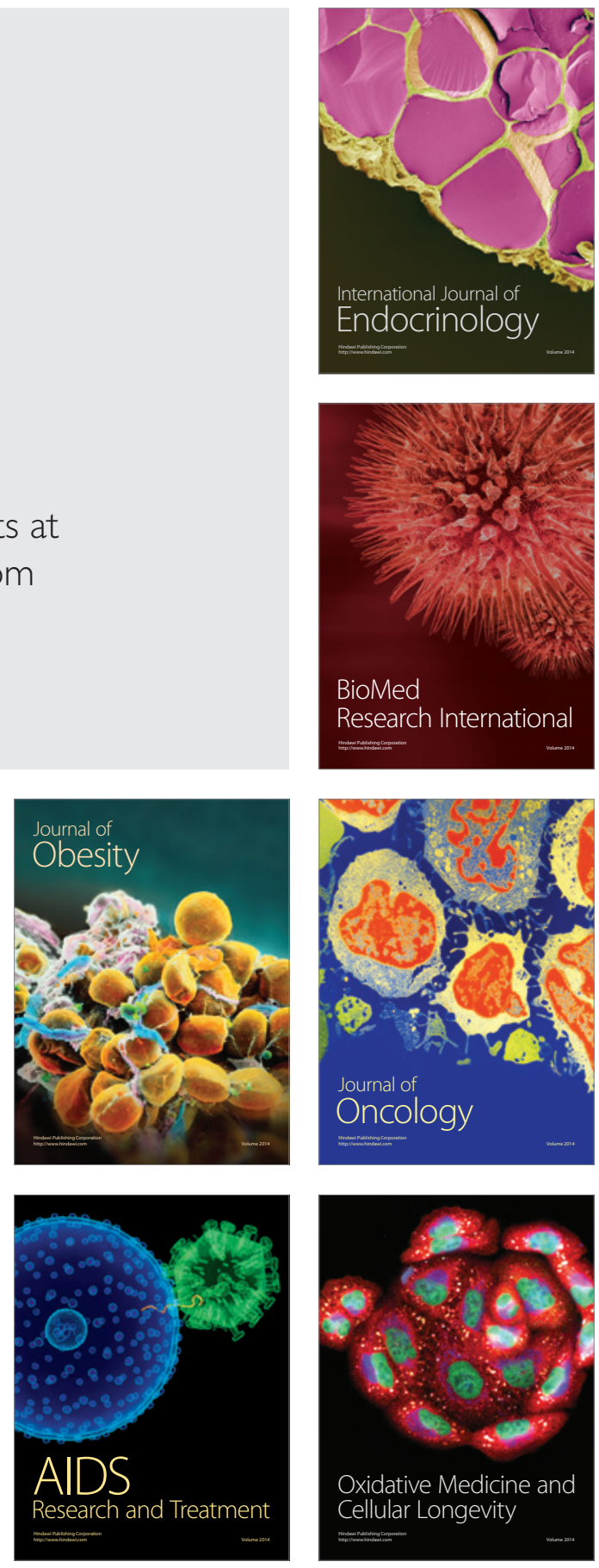